Check for updates

Cite this: RSC Adv., 2019, 9, 52

Received 12th October 2018 Accepted 13th December 2018

DOI: $10.1039 / \mathrm{c} 8 \mathrm{ra0} 8472 \mathrm{f}$

rsc.li/rsc-advances

\section{Sub-lethal hyperthermia promotes epithelial-to- mesenchymal-like transition of breast cancer cells: implication of the synergy between hyperthermia and chemotherapy $\dagger$}

\author{
Tae Hee Lee, (D) $t^{\mathrm{a}}$ Jiyoon Bu, $\$^{\mathrm{b}}$ Byoung Hyuck Kim, ${ }^{\mathrm{c}}$ Michael J. Poellmann, ${ }^{\mathrm{b}}$ \\ Seungpyo Hong*bd and Sung Hee Hyun*a
}

Thermotherapy has demonstrated a potential to be an effective non-surgical technique to treat breast cancer. Despite its advantages, including low toxicity and high repeatability, thermotherapy is typically required to be applied in combination with other treatments since the residual tumor cells that survive after hyperthermal treatment often cause recurrence. In this study, we confirmed that breast cancer cells tolerate temperature of up to $47{ }^{\circ} \mathrm{C}$ by synthesizing a large amount of heat shock proteins. Further changes in the molecular properties of the heat-exposed cells were investigated using western blotting, quantitative reverse transcription polymerase chain reaction, and immunocytochemistry. We found that low-temperature hyperthermia promoted epithelial-to-mesenchymal-like transition (EMT), as observed by the increased mesenchymal marker expression levels while decreasing epithelial markers. Moreover, cell morphology changed from cobblestone-like to a more spindle-like appearance, in addition to significantly enhanced cell motility upon heat treatment. These results all support that sub-lethal hyperthermal stress induces EMT. In addition, we examined changes in the chemo-sensitivity of the heat-treated cells. Addition of a chemo-drugs caused increased cytotoxicity of the heat-treated cells compared to the cells that were not co-treated with heat. Our study demonstrates that thermotherapy alone may cause undesirable EMT, which could be well overcome through a synergistic effect when applied with chemotherapy.

\section{Introduction}

Breast cancer is one of the most common types of cancer, accounting for $29 \%$ of all new cancer cases among females. ${ }^{1}$ Approximately one in eight women is expected to suffer from breast cancer during her lifetime. ${ }^{2}$ Despite increased survival rates due to advances in cancer treatment, breast cancer still remains one of the most fatal diseases that lead to death. ${ }^{1}$ Chemotherapy combined with mastectomy or radiotherapy is currently regarded as a gold standard for treating breast

\footnotetext{
${ }^{a}$ Department of Senior Healthcare, BK21 plus program, Graduated School, Eulji University, 77 Gyeryong-ro, Jung-gu, Daejeon 34824, Republic of Korea. E-mail: hyunsh@eulji.ac.kr; Fax: +82 42-259-1759; Tel: +82 42-259-1751

${ }^{b}$ Division of Pharmaceutical Sciences, School of Pharmacy, University of WisconsinMadison, 777 Highland Ave., Madison, Wisconsin 53705, USA. E-mail: seungpyo. hong@wisc.edu; Fax: +1-608-262-5345; Tel: +1-608-890-0699

'Department of Radiation Oncology, Seoul Metropolitan Government Seoul National University Boramae Medical Center, Seoul 07061, Republic of Korea

${ }^{d}$ Yonsei Frontier Lab, Department of Pharmacy, Yonsei University, Seoul, 03722, Republic of Korea

$\dagger$ Electronic supplementary information (ESI) available. See DOI: $10.1039 / \mathrm{c} 8 \mathrm{ra} 08472 \mathrm{f}$

$\ddagger$ Both authors contributed equally to this work.
}

cancer. ${ }^{3}$ However, dosage of chemo treatment needs to be carefully chosen, given that an unnecessarily high dose of anticancer agents often leads to serious side effects and unexpected complications, such as anesthesia or stroke.

Alternatively, thermotherapy, which eliminates malignant cells by inducing hyperthermal stress at the tumor locations, is one of the most extensively studied non-surgical methods for treating breast cancer. Various types of thermotherapies, including regional hyperthermia, local thermal ablation, and magnetic hyperthermia have been developed to promote necrosis of cancer cells. ${ }^{4}$ The biggest advantage of hyperthermia is that the therapy is tolerable for most patients even at high dose and shows extremely low toxicity. ${ }^{5}$ Moreover, hyperthermia is less invasive compared to other conventional surgical methods and can be administered repeatedly. ${ }^{6}$

Hyperthermia is generally applied in combination with other conventional treatments because the residual tumor cells that survive elevated temperatures have the potential to cause recurrence. ${ }^{7}$ In some case, these residual cancer cells show high invasiveness and proliferative characteristics, making the overall treatment outcome undesirable. Kong et al. have demonstrated that unsuccessful thermal treatment could result 
in angiogenesis of the residual tumor and promote the rapid growth of hepatocellular carcinoma. ${ }^{8}$ Other groups also have suggested that sub-lethal hyperthermia could enhance malignant potentials through a mechanism that promotes epithelialto-mesenchymal transition (EMT). ${ }^{\mathbf{9 - 1 1}}$ Higher levels of hyperthermal stress can mitigate these side effects by causing greater damage to cancer cells. However, considering that hyperthermal stress can also damage normal tissues near the malignant tumor, most of the currently available thermotherapies have shown limited success when applied alone.

In this study, we focused on investigating the effectiveness of hyperthermia as an adjuvant therapy for chemotherapy, particularly at sub-lethal temperature (at or below $47^{\circ} \mathrm{C}$ ). First, we confirmed that the cancer cells are capable of tolerating temperatures up to $47{ }^{\circ} \mathrm{C}$ using two breast cancer cell lines, MCF-7 and MDA-MB-231. The expression of epithelial and mesenchymal features was investigated using western blotting, quantitative reverse-transcription polymerase chain reaction (qRT-PCR), and immunocytochemistry (ICC). We further examined changes in the morphology and migratory capacity of the heat-induced cancer cells to assess whether sub-lethal heat triggers phenotypic changes. Finally, we treated heat-exposed cells with low doses of anticancer agents to confirm that thermotherapies can still be beneficial when combined with chemotherapy. Our results reveal that the synergistic effects of chemotherapy and thermotherapy effectively induce apoptosis or necrosis of cancer cells at relatively low doses of anticancer agents, while preventing EMT-like changes in residual tumor cells and reducing the side effect from each of the therapies.

\section{Materials and methods}

\section{Inducing sub-lethal hyperthermia and viability measurement}

Two human breast cancer cell lines, MCF-7 and MDA-MB-231, were employed in this study. Hyperthermal stress was induced by elevating the temperature of an incubator (CO2CELl50, Revodix, Korea) to $42{ }^{\circ} \mathrm{C}, 47{ }^{\circ} \mathrm{C}$, or $52{ }^{\circ} \mathrm{C}$. After $45 \mathrm{~min}$ of exposure, cells were immediately moved back to an incubator (MCO18AIC, Sanyo, Japan) with temperature set at $37{ }^{\circ} \mathrm{C}$ and maintained for 1-24 h. Stimulation of EMT was accomplished through treatment with TGF- $\beta 1$ (Thermo Scientific, DE), a well-established induction factor. ${ }^{12}$ See ESI $\dagger$ for additional details.

The cell viability was measured using a two-colored live/dead cell viability assay kit (Life Technology, CA), as described elsewhere. ${ }^{13}$ Heat-treated and untreated cells were kept in solution having $4.0 \mu \mathrm{M}$ of Calcein acetoxymethyl and ethidium homodimer, respectively, for $20 \mathrm{~min}$. Cells were then washed with phosphate-buffered saline (PBS) solution.

\section{Cell morphology analysis and migration assay}

The morphological changes of the cells upon sub-lethal heat treatment were first analysed by observing phase contrast images and then verified in more details using periodic acidSchiff (PAS) and crystal violet staining. Cell images were acquired using two different phase-contrast microscopies
(Eclipse Ti-E, Nikon, Japan and Zeiss Axiocam 503 mon, Carl Zeiss, Germany) with a $20 \times$ or $5 \times$ objective.

The migratory behavior of cells following hyperthermic treatment was monitored using the in vitro wound healing assay, as described elsewhere. ${ }^{\mathbf{1 4}}$ Cells were heat-treated and wounds were created by scraping monolayer of cells with a swab. Cell migration was observed after $48 \mathrm{~h}$ of incubation. See ESI $\dagger$ for additional details.

\section{Molecular analysis}

Changes in the molecular properties of the heat-exposed cells were investigated using ICC, western blotting, and qRT-PCR. Five different primary antibodies (DAKO, Denmark), anti-Ecadherin (1:300), anti-CK8/18 (1:200), anti-vimentin $(1: 1000)$, anti-desmin $(1: 150)$, and anti-p53 (1:300), were employed for immunocytochemistry (ICC). The antibodies were applied to quantify the changes in EMT-related properties or demonstrate the cell death mechanism.

The changes in protein expression were further determined by western blotting. The expression of heat shock protein 70 (Hsp70, 1 : 1000, Merck Millipore, Germany), Hsp90 (1: 500, Santa Cruz, CA, USA), E-cadherin (41:1000, Merck Millipore), and vimentin $(1: 1000$, Merck Millipore) were quantified by comparing to that of $\beta$-actin $(1: 1000$, Merck Millipore).

EMT-related genes were quantified using qRT-PCR. Primer sequences, including HSPA1A (Hs00359163_s1), CDH1 (Hs01023894_m1), VIM (Hs00958111_m1), TWIST1 (Hs01675818_s1), and GADPH (Hs02758991_g1), were purchased from Thermo Fisher Scientific. See ESI $\dagger$ for additional details.

\section{Cytotoxicity assay}

Paclitaxel and cisplatin (Sigma) were dissolved in a mixture of dimethyl sulfoxide (DMSO) and methanol (1:9) and stored at $4{ }^{\circ} \mathrm{C}$ before use. Paclitaxel and cisplatin were then resuspended in culture media at a concentration of $10 \mathrm{ng} \mathrm{mL} L^{-1}$ and $1 \mu \mathrm{g}$ $\mathrm{mL}^{-1}$, respectively. The final concentration of DMSO did not exceed $0.05 \%$ in the culture medium.

\section{Results and discussion}

\section{Effect of sub-lethal hyperthermia on cell viability}

To test the tolerability of cells to heat treatment, two human breast cancer cell lines, MCF-7 and MDA-MB-231, were incubated at various temperatures for $45 \mathrm{~min}$. The heat-treatment time, $45 \mathrm{~min}$, was determined after measuring the actual temperature of the cell media for $60 \mathrm{~min}$ (Fig. S1†). We found that the actual temperature increases rapidly for the first $20 \mathrm{~min}$, reaching approximately $90 \%$ of $\Delta T_{\text {target-actual }}$. Considering that the local hyperthermia treatment in generally conducted for 20-60 min at the temperature between $40-45{ }^{\circ} \mathrm{C}$, we can estimate that the cells are exposed to the hyperthermal stress at the desired temperature for at least 25 min. ${ }^{15-17}$

Viability of both cell lines decreased as the exposure temperature increased (Fig. 1A). Viability rates were $92.9 \pm$ $3.1 \%, 89.5 \pm 1.7 \%$, and $81.7 \pm 10.7 \%$ for MCF-7 cells and $90.2 \pm$ 


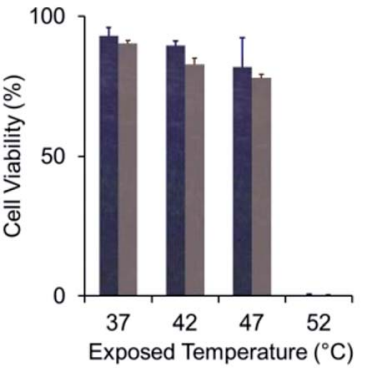

(a)

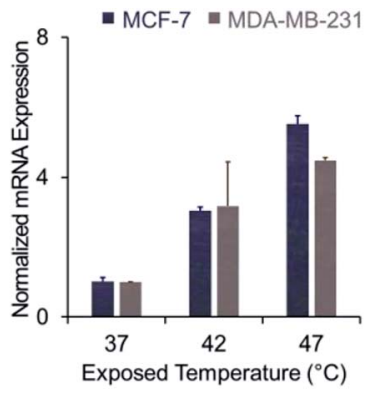

(b)
Fig. 1 Effect of sub-lethal hyperthermal stress on cell viability: (a) cell viability depending on exposed temperature; (b) expression of heat shock protein 70 (Hsp70) after heat treatment.

$1.2 \%, 82.8 \pm 2.2 \%$, and $77.9 \pm 1.2 \%$ for MDA-MB-231 cells, immediately following incubation at $37{ }^{\circ} \mathrm{C}, 42{ }^{\circ} \mathrm{C}$, and $47^{\circ} \mathrm{C}$, respectively. When the temperature was elevated to $52{ }^{\circ} \mathrm{C}$, hyperthermal stress induced cell death a rate greater than $95 \%$. A substantial fraction of cultured breast cancer cells (over 80\%) tolerated hyperthermal treatment at or below $47^{\circ} \mathrm{C}$. The results suggest that cancer cells exposed at $47{ }^{\circ} \mathrm{C}$ would have high potential to survive and cause recurrence.

A number of previous reports have demonstrated that cell apoptosis is induced at $45^{\circ} \mathrm{C}$, and most cells are unable to resist temperatures above $50-55{ }^{\circ} \mathbf{C}^{\mathbf{1 8 , 1 9}}$ Our results are in a good agreement with the existing literature. The high thermotolerance at temperatures below $50{ }^{\circ} \mathrm{C}$ has been attributed to the cells synthesizing heat shock proteins, which stabilize and repair denatured proteins and help to transfer secretory proteins. ${ }^{20}$ These proteins are also involved in cell proliferation and are known to be overexpressed in cancer cells. ${ }^{21} \mathrm{We}$ analysed the mRNA expression of Hsp70, one of the most welldefined molecular chaperones that protect cells from thermal stress (Fig. 1B). Expression of Hsp70 was enhanced by 3.0-folds in MCF-7 ( $p=0.013)$ and 3.2-folds in MDA-MB-231 ( $p=0.125)$, when the temperature was elevated to $42{ }^{\circ} \mathrm{C}$. The results were more significant at $47^{\circ} \mathrm{C}$, showing 5.5-fold $(p=0.006)$ and 4.5fold enhancement $(p<0.001)$ for MCF-7 and MDA-MB-231, respectively. Additionally, we measured the expression levels of Hsp70 and Hsp90 $\alpha$ (another heat shock protein) proteins from the MDA-MB-231 cell line using polyacrylamide gel electrophoresis, shown in Fig. S1. $\dagger$ These results showed that cancer cells synthesize increasing amounts heat shock proteins in response to increasing levels of hyperthermal stress. Our data indicate that cancer cells protect themselves by synthesizing these molecular chaperones.

\section{Effect of sub-lethal hyperthermia on cell morphology and migratory capacity}

We first investigated the changes in cell morphology by comparing the bright-field images, before and after heat treatment. Altered cellular morphology is commonly reported following heat treatment. As shown in Fig. 2A, the cells changed from cobblestone-like to a more spindle-like appearance after heat treatment. A similar pattern was observed when the cells were treated with TGF- $\beta 1$ (Fig. S2 $\dagger$ ). Moreover, cell-to-cell contact was decreased in heat-treated cells, which may also be evidence of EMT. We further conducted PAS and H\&E staining to confirm the morphological changes in more detail. As shown in Fig. 2B and $\mathrm{S} 3, \dagger$ the stain revealed pseudopodial protrusions typical of fibroblast-like cells, which is also observed after TGF$\beta 1$ treatment. Therefore, we have found that the cancer cells exposed to low-temperature hyperthermia demonstrated similar morphological changes with the cells that experienced TGF- $\beta 1$-induced EMT.

Next, we conducted a cell migration assay to further elucidate whether the sub-lethal heat treatment could facilitate functional changes. Cells were scraped from the dish following hyperthermia in an in vitro wound model. Heat treatment at $42{ }^{\circ} \mathrm{C}$ (Fig. 2C) stimulated greater migration in both cell lines. The migratory capacity was enhanced by 1.57 -fold for MCF-7 ( $p$ $=0.009)$ and 2.42-fold for MDA-MB-231 cells $(p=0.016)$. The enhancement was comparable to the cells that were incubated with TGF- $\beta 1$, which showed 1.47 -fold $(p=0.032)$ and 1.86 -fold $(p<0.001)$ enhancement for MCF-7 and MDA-MB-231, respectively. Cells treated at $47{ }^{\circ} \mathrm{C}$ had differing effects. MCF-7 cells still showed higher migratory capacity (1.25-fold enhancement with $p=0.084$ ), but their migratory capacity was lower than that of cells kept at $42{ }^{\circ} \mathrm{C}$. In contrast, the migratory capacity of MDAMB-231 cells was reduced by $30 \%(p=0.049)$ compared to controls treated at $37{ }^{\circ} \mathrm{C}$.

The reduction in MDA-MB-231 migration after $47{ }^{\circ} \mathrm{C}$ treatment may be attributed to cell death. In the previous section, cell viability was measured immediately after inducing hyperthermal stress. We incubated cells for $3 \mathrm{~h}$ at $37{ }^{\circ} \mathrm{C}$ after heat treatment and re-analysed the cell viability (Fig. 2D). There were no significant differences in cell viability, when the temperature was elevated up to $42{ }^{\circ} \mathrm{C}$. Cell viability was reduced from $89.5 \pm$ $1.7 \%$ to $86.3 \pm 0.7 \%$ for MCF-7 cells and from $82.8 \pm 2.2 \%$ to $80.2 \pm 6.2 \%$ for MDA-MB-231 cells after $3 \mathrm{~h}$ of incubation. Cell viability reduction was still not strongly pronounced for MCF-7 cells, even after exposing them at $47{ }^{\circ} \mathrm{C}(81.7 \pm 10.7 \%$ and 80.5 $\pm 9.4 \%$ when measured immediately and after $3 \mathrm{~h}$ of incubation, respectively). However, MDA-MB-231 cells showed significant cell viability reduction, which decreased from $77.9 \pm 1.2 \%$ to $61.6 \pm 7.3 \%$. We extended the incubation time to $6 \mathrm{~h}$ and found out that there was no further reduction in cell viability $(65.0 \pm 12.3 \%$ after $6 \mathrm{~h}$ of incubation). Although more than $60 \%$ of the cells were still capable of surviving from heat exposure, there was a significant decrease in the viability of MDA-MB-231 cells. Elengoe et al. have shown that, despite being moderately different, MDA-MB-231 cells demonstrated higher heat sensitivity compared to MCF-7 cells. ${ }^{22,23}$ Therefore, low-temperature thermotherapy has possibility to mediate functional and morphological changes to the cells that survive from the hyperthermal stress.

\section{Effect of sub-lethal hyperthermia on phenotypic properties}

We measured the expression of EMT-related markers to further evaluate the effects of hyperthermal stress on cellular 


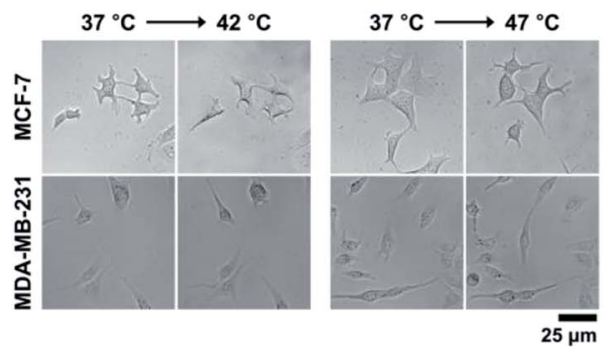

(a)

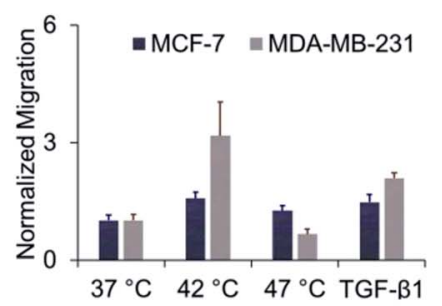

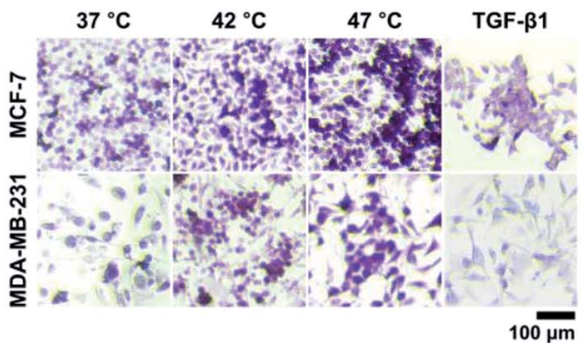

(b)
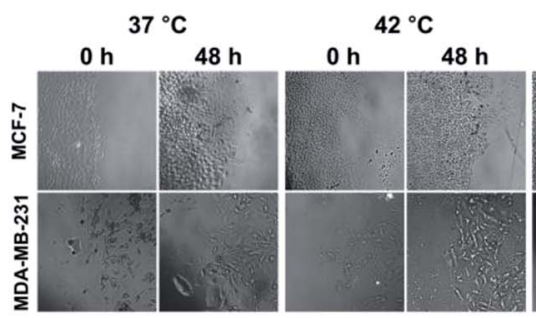

(c)

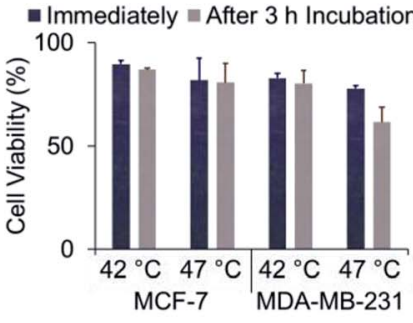

(d)

Fig. 2 Effect of sub-lethal hyperthermal stress on cell morphology and migratory capacity: (a) bright field images and (b) periodic acid Schiff (PAS)-stained images of cells after sub-lethal hyperthermia treatment; (c) and migratory capacity of cells after sub-lethal hyperthermia or TGF- $\beta 1$ treatment; (d) viability of heat-treated cells after $3 \mathrm{~h}$ of incubation.

phenotype. The results of western blot analysis are shown in Fig. 3A. The expression level of an epithelial marker, Ecadherin, was significantly downregulated in MCF-7 cells following thermal stress. No significant changes were found in MDA-MB-231 cells, which have low expression of E-cadherin even without heat treatment. In contrast, a mesenchymal marker, vimentin, was upregulated in both cell lines.

Similarly, mRNA expression analysis demonstrated increased mesenchymal marker expression whereas the epithelial marker expression was decreased (Fig. 3B). Changes in the expression of the EMT-related markers after heat treatment were not as significant as those induced by TGF- $\beta 1$ treatment, although the trend was similar. The expression of E- cadherin in MCF-7 cells was reduced by $85.9 \%(p=0.437)$ and $70.7 \%(p=0.042)$ following hyperthermic treatment at $42{ }^{\circ} \mathrm{C}$ and $47^{\circ} \mathrm{C}$, respectively. The TGF- $\beta 1$ treatment similarly resulted in lower E-cadherin expression $(p=0.064)$. E-cadherin expression could not be detected in MDA-MB-231 cells following any treatments. Upregulation of vimentin in response to hyperthermia was significant in both cell lines, showing 1.13-fold ( $p=$ $0.130)$ and 1.76-fold $(p=0.018)$ enhancement for MCF-7 cells and 1.42-fold $(p=0.038)$ and 2.89-fold $(p=0.008)$ enhancement for MDA-MB-231 cells, upon heat treatment at $42{ }^{\circ} \mathrm{C}$ and $47^{\circ} \mathrm{C}$, respectively. The TGF- $\beta 1$ treatment enhanced vimentin expression by 3.11-fold and 6.25-fold for MCF-7 cells and MDA-MB-231 cells, respectively.

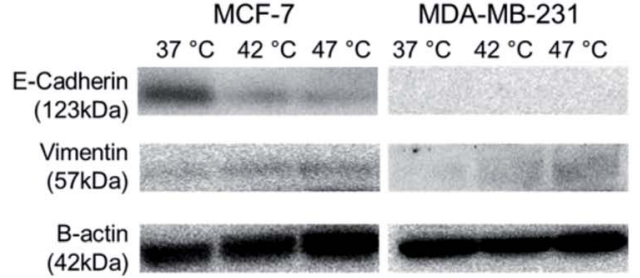

(a)

Relative mRNA Expression

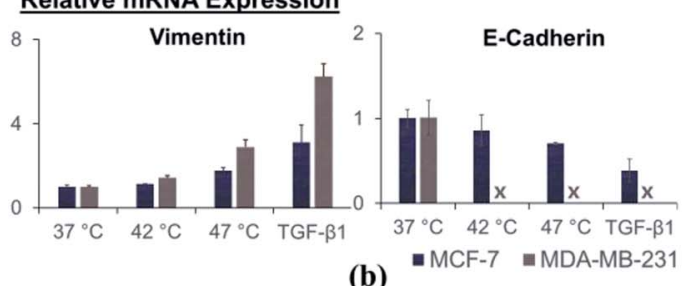

(b)

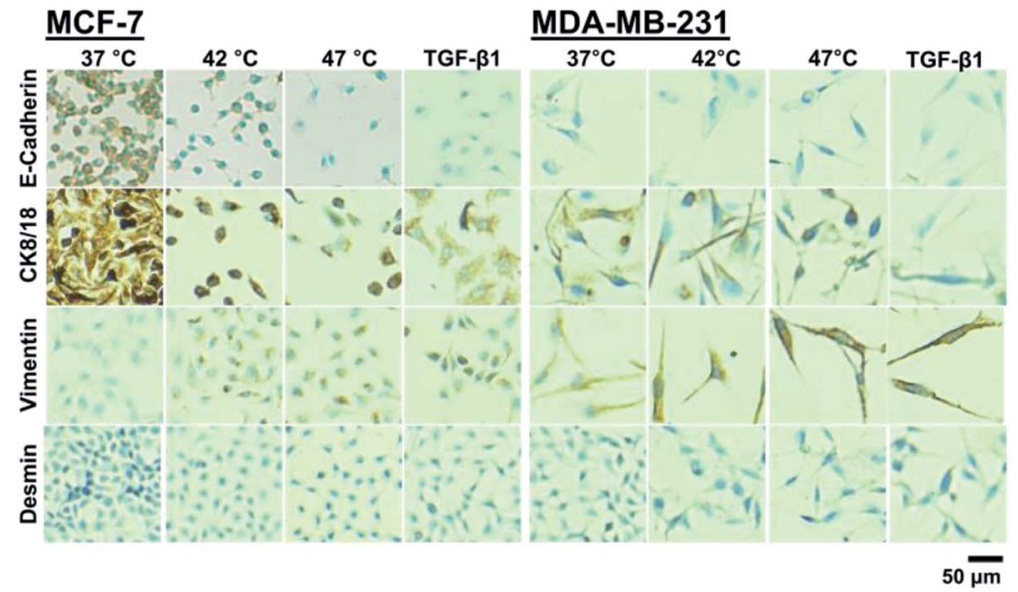

(c)

Fig. 3 Effect of sub-lethal hyperthermia on changes in expression level of EMT-related markers, analyzed by (a) western blot assay, (b) qRT-PCR, and (c) immunocytochemistry. 
Immunocytochemical staining further demonstrated the suppression of epithelial markers E-cadherin and CK8/18, and the enhancement of mesenchymal markers vimentin and desmin (Fig. 3C). We observed reduced epithelial marker expressions in cytoplasm or cell membrane after heat treatment, and enhanced expression for mesenchymal markers. In addition, immunocytochemical stained cells were elongated and spindleshaped, consistent with EMT.

Recently, a number of researchers have reported links between EMT and sub-lethal cancer treatment. Yoshida et al. observed the upregulation of EMT-activating transcription factors, Twist1 and Snail, after exposing hepatocellular carcinoma cell lines to sublethal hyperthermal condition. ${ }^{18}$ Dong et al. verified that low-temperature heat treatment promoted EMT in hepatocellular carcinoma cells via Akt and ERK signaling pathways and detected increased Snail expression. ${ }^{24}$ We also examined the enhanced expression of two different transcription factors, Snail and Twist1, after heat-treating breast cancer cell lines. The relative mRNA expression level was slightly increased following sub-lethal heat treatment (Fig. S4†). Although changes in expression were lower in magnitude than those observed with TGF- $\beta 1$ treatment, our data strongly suggest that EMT is promoted in hyperthermia.

\section{Implication of the synergy between hyperthermia and chemotherapy}

Anticancer agents were applied after exposing cells to hyperthermal conditions (Fig. 4 and S5†). Without any heat treatment, chemotherapy induced only $18.7 \pm 5.4 \%, 30.0 \pm 7.9 \%$, and $34.2 \pm 9.9 \%$ cell death in MCF-7 cultures treated with

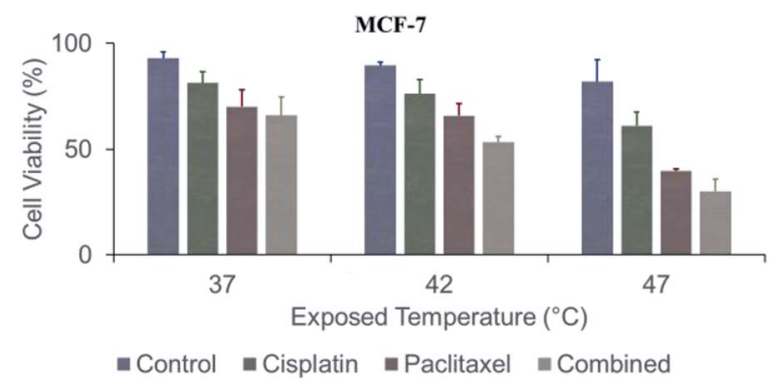

(a)

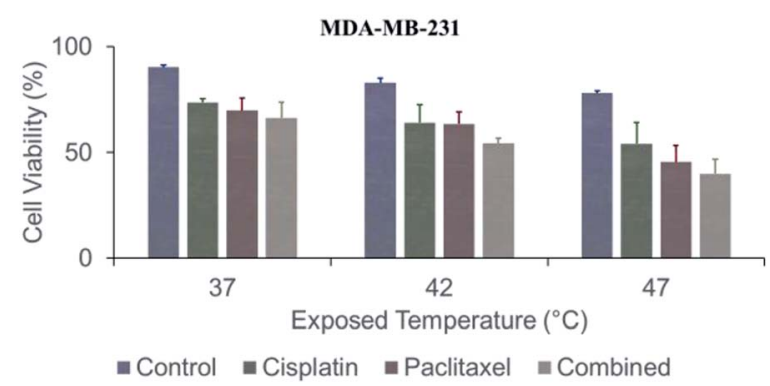

(b)

Fig. 4 Effect of sub-lethal hyperthermia on chemo-sensitivity: (a) viability of MCF-7 cells after thermo- and/or chemotherapy; (b) viability of MDA-MB-231 cells after thermo- and/or chemo-therapy. cisplatin, paclitaxel, and the combination of the two agents, respectively. Similarly, $26.5 \pm 1.9 \%, 30.4 \pm 5.9 \%$, and $33.6 \pm$ $7.3 \%$ of MDA-MB-231 cells were killed following treatment with the same anticancer agents. These results confirmed that hyperthermia is a powerful adjuvant treatment for chemotherapy in cultured cells. The combined effect of chemotherapy and thermotherapy at $42{ }^{\circ} \mathrm{C}$ resulted in $23.9 \pm 6.2 \%(p=0.179)$, $34.3 \pm 6.5 \%(p=0.406)$, and $73.7 \pm 5.4 \%(p=0.088)$ of MCF-7 cell death, which were higher than when cisplatin, paclitaxel, and the combination of the two agents used alone, respectively. A further increase in the synergistic effect was observed at $47^{\circ} \mathrm{C}$. Upon co-treatment with cisplatin, paclitaxel, and the combination of the two agents, $38.8 \pm 6.3 \%(p=0.003), 69.9 \pm 5.7 \%(p$ $<0.001)$, and $73.7 \pm 5.4 \%(p=0.001)$ of the MCF-7 cells lost viability, respectively. Similarly, chemo-sensitivity was enhanced after treating MDA-MB-231 cells at $42{ }^{\circ} \mathrm{C}$, causing $35.9 \pm 8.3 \%$ (cisplatin; $p=0.062$ ), $36.4 \pm 5.5 \%$ (paclitaxel; $p=$ 0.158 ), and $46.1 \pm 2.5 \%$ (combined; $p=0.036$ ) cell death. Hyperthermal treatment at $47{ }^{\circ} \mathrm{C}$ significantly enhanced the chemo-sensitivity by inducing $45.8 \pm 8.3 \%$ (cisplatin; $p=$ 0.030 ), $54.6 \pm 7.8 \%$ (paclitaxel; $p=0.001$ ), and $60.1 \pm 6.9 \%$ (combined; $p=0.001$ ) of the cell death. We concluded that the hyperthermal stress aided the anticancer agents in promoting cell death.

We further explored the effect of combined treatment by analyzing the cells with DAPI and p53 staining (Materials and methods section in ESI and Fig. S4 $\dagger$ ) to confirm apoptotic and necrotic cell death. Crystal violet staining was also conducted to observe colony formation after thermotherapy and/or chemotherapy. The cancer cells initially had intact nuclear structures, adopting a more rounded, mitotic-like appearance with multiple nuclei in a cell after combined treatment. We additionally observed that the p53 tumor suppressor, which activates apoptosis, was slightly activated as a consequence of the synergistic effect of thermotherapy and chemotherapy. ${ }^{25}$ The combined therapy additionally resulted in reduced colony formation with growth inhibition accompanied by membrane shrinkage and rounded appearance. Therefore, the combined use of thermotherapy and chemotherapy appeared to promote cancer cell death even at inadequate doses of anticancer agents.

Although hyperthermia could show the undesired outcomes when it is conducted alone, it could be highly synergistic when combined with chemotherapy because of the following reasons. First, despite the small effect of sub-lethal heat treatment on cell viability, hyperthermia could still promote apoptosis of the cells that are non- or less-sensitive to the specific chemotherapeutic agents. We have demonstrated throughout our study that the low-temperature hyperthermal stress still induces $10-25 \%$ of cancer cell death. Second, heat treatment enhances cell perfusion, facilitating therapeutic agents to diffuse into cell membrane. ${ }^{26}$ The increase in membrane permeability via hyperthermal stress allows more therapeutic agents to enter through the cell membrane. Third, the change in cell morphology, cobblestone-like to more spindle like appearance, could also affect the absorption of therapeutic agents. The morphological change increases surface-area-to-volume ratio of the cells, providing higher possibility for the cancer cells to 
absorb more chemo-drugs. Finally, sub-lethal heat treatment affects the DNA repairing mechanism, promoting more cancer cell death after chemotherapy. ${ }^{27}$

\section{Conclusions}

In this study, our results indicate that low-temperature hyperthermia enhanced the mesenchymal-like characteristics of the residual tumor. Over $75 \%$ of the breast cancer cells were capable of tolerating temperatures below $47{ }^{\circ} \mathrm{C}$ by synthesizing heat shock proteins. The enhanced motility and morphological changes from cobblestone-like to a more spindle-like appearance also indicate that cells have undergone EMT-like changes after low-temperature hyperthermia. Moreover, qRT-PCR, western blotting, and ICC analyses all supported that sublethal hyperthermia result in EMT-like changes by showing increased mesenchymal marker expression while decreasing epithelial markers. Although sub-lethal hyperthermal stress induced EMT-like changes in cultured cells, hyperthermia showed remarkable synergistic effects with chemotherapy. The co-treatment of hyperthermia with low doses of chemotherapeutic agents exhibited more than doubled cytotoxic effect to the cancer cells, compared to the individual treatments with chemo-drugs only. Our data confirm the synergistic effect of the chemotherapy-thermotherapy combination, supporting that the potential benefit of such co-treatment in treating cancer. For the future work, we are planning to extend our study using mouse models and confirm whether this phenomenon still occurs in vivo. Then, we will investigate more deeply on finding the mechanism that induces EMT-like changes and enhances chemosensitivity.

\section{Conflicts of interest}

There are no conflicts to declare.

\section{Acknowledgements}

This study was partially supported by the National Science Foundation (NSF) under grant \# DMR-1409161/1741560, awarded to $\mathrm{SH}$.

\section{Notes and references}

1 A. Jemal, F. Bray, M. M. Center, J. Ferlay, E. Ward and D. Forman, Ca-Cancer J. Clin., 2011, 61, 69-90.

2 E. Alphandéry, Cancer J., 2014, 5, 472-479.

3 V. Kouloulias, S. Triantopoulou, N. Uzunoglou, K. PistevouGompaki, A. Barich, A. Zygogianni, G. Kyrgias, D. Kardamakis, D. Pectasidis, J. Kouvaris and G. S. o. H. Oncology, Breast Care, 2015, 10, 109-116.
4 Y. Zhou, L. Bao and C. L. P. Chen, Signal Process., 2013, 93(11), 3039-3052.

5 A. Kariya, Y. Furusawa, T. Yunoki, T. Kondo and Y. Tabuchi, Int. J. Mol. Med., 2014, 34, 334-340.

6 W. Y. Lau and E. C. Lai, Ann. Surg., 2009, 249, 20-25.

7 H. Rhim, H. K. Lim, Y. S. Kim and D. Choi, AJR, Am. J. Roentgenol., 2008, 190, 91-98.

8 J. Kong, B. Pan, S. Ke, S. Dong, X. Li, A. Zhou, L. Zheng and W. B. Sun, PLoS One, 2012, 7, e37266.

9 N. Zhang, L. Wang, Z. T. Chai, Z. M. Zhu, X. D. Zhu, D. N. Ma, Q. B. Zhang, Y. M. Zhao, M. Wang, J. Y. Ao, Z. G. Ren, D. M. Gao, H. C. Sun and Z. Y. Tang, PLoS One, 2014, 9, e115949.

10 S. Ke, X. M. Ding, J. Kong, J. Gao, S. H. Wang, Y. Cheng and W. B. Sun, J. Transl. Med., 2010, 8, 73.

11 J. Bu, T. H. Lee, I. S. Kim and Y.-H. Cho, Sens. Actuators, B, 2017, 244, 591-598.

12 J. Bu, Y.-H. Cho and S.-W. Han, RSC Adv., 2017, 7, 4968449693.

13 K. Yoonji Kim, J. Bu, Y. Ho Cho, I. Tae Son and S. Bum, J. Micromech. Microeng., 2017, 27, 025015.

14 W.-C. Chiu, T.-J. Chiou and A.-N. Chiang, Biochem. J., 2012, 445, 125.

15 Z. Zoul, S. Filip, B. Melichar, J. Dvorák, K. Odrázka and J. Petera, Onkologie, 2004, 27, 385-388.

16 R. J. Griffin, R. P. Dings, A. Jamshidi-Parsian and C. W. Song, Int. J. Hyperthermia, 2010, 26, 256-263.

17 A. De Marco and M. G. Santoro, J. Gen. Virol., 1993, 74(Pt 8), 1685-1690.

18 S. Yoshida, M. Kornek, N. Ikenaga, M. Schmelzle, R. Masuzaki, E. Csizmadia, Y. Wu, S. C. Robson and D. Schuppan, Hepatology, 2013, 58, 1667-1680.

19 R. W. Habash, R. Bansal, D. Krewski and H. T. Alhafid, Crit. Rev. Bioeng., 2006, 34, 491-542.

20 J. Verghese, J. Abrams, Y. Wang and K. A. Morano, Microbiol. Mol. Biol. Rev., 2012, 76, 115-158.

21 S. K. Calderwood, M. A. Khaleque, D. B. Sawyer and D. R. Ciocca, Trends Biochem. Sci., 2006, 31, 164-172.

22 A. Elengoe and S. Hamdan, Heat Sensitivity between Human Normal Liver (WRL-68) and Breast Cancer (MCF-7) Cell Lines, 2013.

23 A. Elengoe and S. Hamdan, Heat Sensitivity between Human Normal Liver (WRL-68) and Breast Cancer (MDA-MB 231) Cell lines, 2013.

24 S. Dong, J. Kong, F. Kong, J. Gao, S. Ke, S. Wang, X. Ding, W. Sun and L. Zheng, J. Transl. Med., 2013, 11, 273.

25 J. S. Fridman and S. W. Lowe, Oncogene, 2003, 22, 9030-9040. 26 S. Jha, P. K. Sharma and R. Malviya, Achievements in the Life Sciences, 2016, 10, 161-167.

27 G. G. Jonsson, G. Eriksson and R. W. Pero, Radiat. Res., 1984, 97, 97-107. 